\title{
Terapêutica farmacológica na pré-diabetes
}

António Macedo, ${ }^{*}$ Carla Campos, ${ }^{* *}$ Joana Correia,** Paula Gomes***

\section{RESUMO}

Objectivos: A diabetes mellitus tipo 2 (DM2) é um problema de saúde associado a aumento da morbimortalidade e custos. É frequentemente precedida por estados metabólicos alterados, alteração da glicemia em jejum (AGJ) e diminuição da tolerância à glicose (DTG). Está demonstrado que alterações do estilo de vida em doentes com DTG diminuem a sua incidência. $O$ uso de alguns fármacos parece prevenir ou atrasar a progressão da doença, mas existem dúvidas sobre o seu real benefício. Pretende-se determinar a evidência de benefícios para o doente com a terapia farmacológica nos estados pré-diabéticos.

Fontes de dados: Foi realizada uma pesquisa sistemática, na National Guideline Clearinghouse, Bandolier, DARE, NHS e Medline, de artigos publicados entre Janeiro de 1998 e Setembro de 2008, em língua portuguesa, inglesa e espanhola, usando as palavras «prediabetic state», «prediabetes» e «therapeutics».

Métodos de revisão: Obtiveram-se 103 artigos que comparavam a intervenção farmacológica com placebo ou alterações de estilo de vida em doentes pré-diabéticos. Utilizou-se a classificação SORT para atribuição do nível de evidência e força de recomendação.

Resultados: Foram incluídos 9 artigos: 4 normas de orientação clínica, 1 meta-análise, 3 revisões sistemáticas e 1 ensaio controlado aleatorizado. Alguns antidiabéticos orais (metformina, acarbose e glitazonas) e o orlistat mostraram uma redução no risco de desenvolvimento de diabetes em doentes pré-diabéticos, ficando por esclarecer se esse benefício se mantém após a sua suspensão. A acarbose mostrou diminuir a progressão do espessamento da íntima das carótidas.

Conclusões: Apesar de alguns antidiabéticos orais e o orlistat terem demonstrado diminuir o risco de desenvolvimento de diabetes em doentes pré-diabéticos, não há evidência que demonstre haver benefícios orientados para o doente na sua utilização, uma vez que nenhum dos estudos avaliou como endpoint primário a morbimortalidade e seu custo-eficácia. Encontram-se em curso alguns estudos que pretendem esclarecer estes pontos.

Palavras-chave: Diabetes Mellitus Tipo 2; Estado Pré-diabético; Terapêutica.

\section{INTRODUÇÃO}

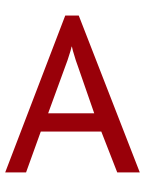

A diabetes mellitus tipo 2 (DM2) é considerada um grave problema de saúde mundial. Estimativas indicam que, no ano 2000, aproximadamente 171 milhões de pessoas com mais de 20 anos de idade tiveram, em todo o mundo, um diagnóstico de diabetes mellitus tipo 2. Com o crescimento e envelhecimento da população, urbanização e aumento da prevalência da obesidade e sedentarismo, as projecções indicam que, em 2030, esta prevalência irá aumentar para mais do dobro. ${ }^{1}$ Em Portugal foi avaliado que, na faixa etária dos 20 aos 79 anos de idade, a prevalência de diabetes é de $11,7 \%$ e que, com pré-diabetes, foi encontrada uma percentagem de 23,2 , corres-

* Interno de Medicina Geral e Familiar no C.S. Senhora da Hora

**Interna de Medicina Geral e Familiar no C.S. de Leça da Palmeira

***Interna de Medicina Geral e Familiar no C.S. de S. Mamede de Infesta pondendo a um número cumulativo de 2.687.698 indivíduos afectados. ${ }^{2}$ Esta é uma doença crónica associada a várias complicações e com consequências graves (retinopatia que pode levar à cegueira, nefropatia que pode conduzir à insuficiência renal terminal, neuropatia periférica, com o risco subjacente de formação de úlceras e, ultimamente, amputação de membro, bem como aumento do risco de doença cardiovascular e cerebrovascular). Está, por isso, associada a uma elevada mortalidade, que a coloca na quinta posição nas principais causas de morte, com um excesso de cerca de 2,9 milhões de mortes atribuíveis à doença. ${ }^{3}$

Vários factores de risco estão implicados no desenvolvimento de diabetes (história familiar, obesidade, hipertensão arterial, dislipidemia, sedentarismo e seguimento de uma dieta não saudável), sendo alvo de atenção e modificação em consultas de promoção da 
QUADRO I. Critérios de diagnóstico dos estádios de metabolismo anormal da glicose

Alteração da Glicemia em Jejum (AGJ) Diminuição da Tolerância à Glicose Oral (DTG)

saúde e prevenção primária. Antes do diagnóstico clínico e analítico da diabetes é possível identificar indivíduos com um elevado risco de desenvolverem a doença pela detecção de um metabolismo anormal da glicose ou insulinoresistência (Quadro I). Este grupo, por vezes referenciado como pré-diabetes, inclui pessoas com alteração da glicemia em jejum (AGJ) ou com diminuição da tolerância à glicose oral (DTG), que apresentam um risco acrescido de progressão para diabetes mellitus tipo 2 bem como um aumento de outros eventos adversos, nomeadamente cardiovasculares. ${ }^{4,5}$ Vários estudos demonstraram que a implementação intensiva de alterações de estilo de vida, com programas regulares de exercício físico e alterações na dieta, permite uma redução significativa da percentagem de indivíduos que irão progredir para diabetes, obtendo ganhos consideráveis de saúde (aproximadamente 58\% de redução em 3 anos). ${ }^{6-10}$ No entanto, é bem conhecida a dificuldade de implementação destas medidas que, pela sua intensidade e regularidade, necessitam da existência de uma elevada motivação e perseverança para serem eficazes. Dada esta dificuldade, outros estudos abordaram a utilização de terapêutica farmacológica na pré-diabetes como forma de diminuir a incidência da doença. ${ }^{6,11-13}$ Verificou-se que alguns fármacos reduziam em graus variáveis a incidência de diabetes; no entanto, a qualidade dos estudos, os problemas do custo, efeitos secundários e falta de um efeito persistente colocam a dúvida sobre a utilização regular da terapêutica farmacológica na pré-diabetes. ${ }^{14}$

Deste modo, é objectivo desta revisão determinar se existem benefícios para o doente no uso de terapia farmacológica nos estados pré-diabéticos.

\section{MÉTODOS}

Foi realizada uma pesquisa sistemática nas bases de dados da National Guideline Clearinghouse, Bandolier, DARE, NHS e Medline, utilizando as palavras-chave prediabetic state, prediabetes e therapeutics, de normas de orientação clínica (NOC), meta-análises (MA), revisões sistemáticas (RS) e ensaios aleatorizados e controlados (EAC), publicados entre 1 de Janeiro de 1998 e 30 de Setembro de 2008, nas línguas portuguesa, inglesa e espanhola.

Os critérios utilizados para inclusão de artigos nesta revisão foram os seguintes:

- População: pessoas com alteração do metabolismo da glicose, isto é, que apresentam alteração da glicemia em jejum ou diminuição da tolerância à glicose oral;

- Intervenção e comparação: intervenção farmacológica com antidiabéticos orais, medicamentos anti-obesidade ou anti-hipertensores versus placebo ou alterações do estilo de vida (como dieta ou programas de exercício físico);

- Resultados: diminuição da mortalidade, diminuição da morbilidade (como diminuição das complicações da diabetes ou diminuição de eventos cardiovasculares) e alteração de parâmetros relacionados com a doença.

Da pesquisa inicial foram obtidos 103 artigos, excluindo-se da análise para esta revisão os artigos repetidos, os artigos que não cumpriam os critérios de inclusão e os estudos originais que foram alvo de análise em NOC, MA e RS incluídas nesta revisão.

Para avaliar o nível de evidência e a força das recomendações foi utilizada a escala Strength of Recommendation Taxonomy (SORT) da American Family Physician. ${ }^{15}$

\section{RESULTADOS}

Após aplicação dos critérios de inclusão e exclusão, obtiveram-se 9 artigos para análise (4 NOC, 1 MA, 3 RS e 1 EAC), expondo-se, de seguida, os resultados encontrados.

Os antidiabéticos orais (metformina, acarbose, troglitazona, rosiglitazona, pioglitazona, tolbutamida, gliclazida), o orlistat e o ramipril foram avaliados em estudos que utilizaram como endpoint primário a incidência de DM2 em doentes com DTG e/ou AGJ.

As principais características, resultados e nível de evidência das NOC, MA, RS e EAC encontrados estão sumariados nos Quadros II, III, IV e V. 


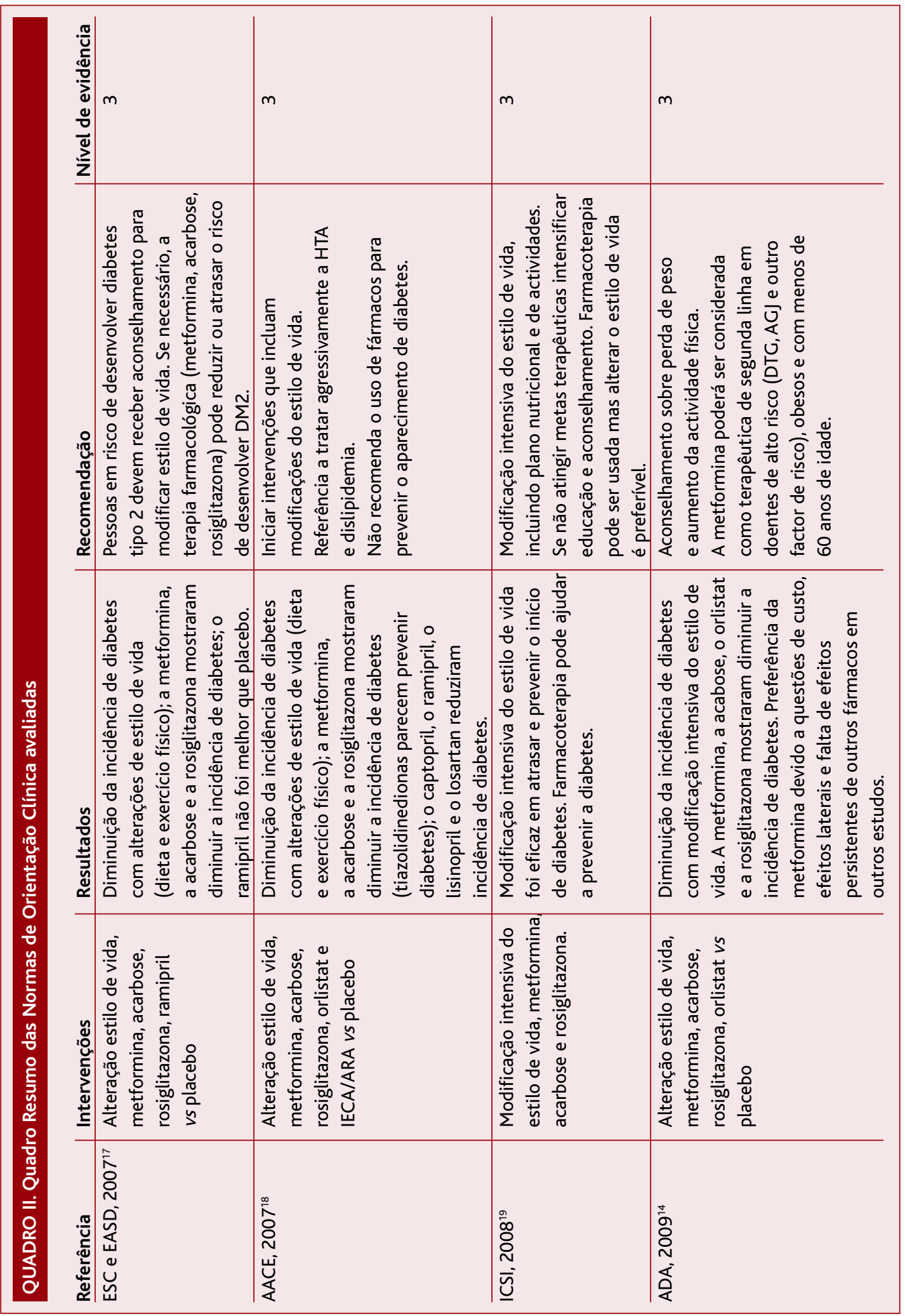




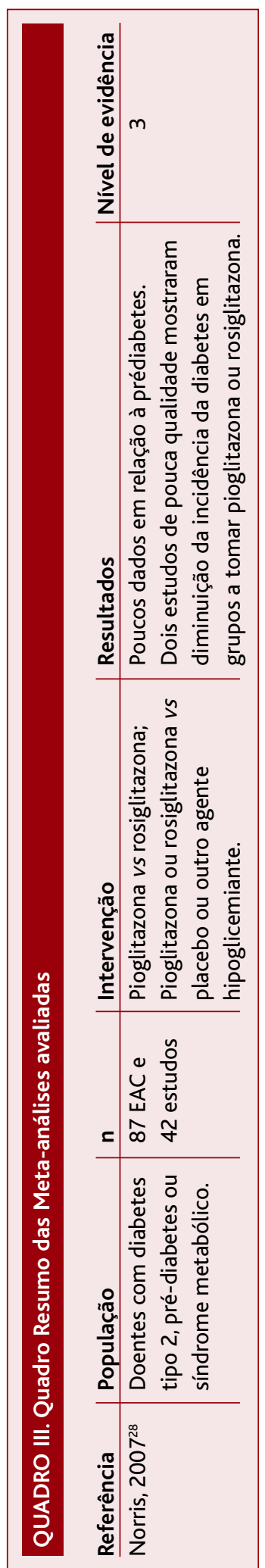

\section{Metformina}

A metformina é a única biguanida em uso clínico actual. Actua reduzindo a produção hepática de glicose e estimulando a captação de glicose pelos tecidos periféricos. ${ }^{16}$

Foram encontradas 4 NOC e 2 RS que avaliaram a utilização da metformina em doentes pré-diabéticos.

A NOC da European Society of Cardiologye European Association for the Study of Diabetes (ESC e EASD), no que concerne à prevenção da progressão para DM2, recomenda que doentes com elevado risco de DM2 devam receber aconselhamento para modificar os estilos de vida e, se necessário, terapêutica farmacológica, incluindo a metformina. ${ }^{17} \mathrm{~A}$ base da recomendação centra-se na comparação dos resultados obtidos com as intervenções no estilo de vida na redução do risco relativo (RRR) de progressão para DM2 relativamente à terapêutica farmacológica. As modificações do estilo de vida resultaram em RRR de 46 a $63 \%$ em estudos com follow-up de 3 a 6 anos, enquanto que a utilização da metformina levou a RRR entre $22 \mathrm{e} 31 \%$ em estudos com follow-up de 2,8 a 3 anos. ${ }^{17}$

A American Diabetes Association (ADA) recomenda que doentes com DTG ou AGJ devam receber, como terapêutica de primeira li- nha, aconselhamento sobre perda de peso ( 5 a $10 \%$ do peso corporal) e aumento da actividade física (150 minutos por semana de actividade moderada). Relativamente à terapêutica farmacológica, apenas recomenda a utilização de metformina e, apenas em indivíduos de risco muito elevado (DTG e AGJ associadas a outro factor de risco), obesos e com menos de 60 anos de idade. À semelhança da ESC e EASD, a recomendação baseou-se na comparação dos resultados das intervenções no estilo de vida versus terapêutica farmacológica. A restrição a indivíduos obesos e com menos de 60 anos fundamenta-se nos resultados do Diabetes Prevention Program (DPP), no qual a metformina apresentou maior eficácia na RRR da progressão para DM2 nesse sub-grupo. ${ }^{14}$

O Institute for Clinical Systems Improvement (ICSI), baseado nos mesmos estudos, preconiza intervenções centradas nas modificações do estilo de vida e redução do risco cardiovascular em detrimento da terapêutica farmacológica. ${ }^{18}$ A American Association of Clinical Endocrinologists (AACE) não recomenda o uso de fármacos para prevenir o aparecimento de diabetes. ${ }^{19}$

Em relação às RS, Padwal et al realizaram uma revisão da terapêutica farmacológica para a prevenção ou atraso no aparecimento da DM2. ${ }^{20}$ Quanto à metformina, incluíram quatro EAC.

O estudo de maior tamanho amostral e maior rigor metodológico foi o DPP, que randomizou 2.155 doentes com DTG para tratamento com metformina $(850 \mathrm{mg}$ b.i.d) ou placebo (um outro braço do estudo avaliou a eficácia das intervenções no estilo de vida e outro braço avaliou a troglitazona; ver abaixo). Após um follow-up de 2,8 anos, a incidência de DM2 foi de 7,8\% no grupo placebo e $4,8 \%$ no grupo tratado com metformina, obtendo-se uma RRR de progressão para DM2 de $31 \%$. Os benefícios da metformina foram primariamente observados em doentes com menos de 60 anos (RRR de 34\% em doentes com 25-44 anos) e com Índice de Massa Corporal (IMC) $\geq 35 \mathrm{~kg} / \mathrm{m}^{2}$ (RRR de 53\%). Neste mesmo estudo, as intervenções no estilo de vida estiveram associadas a RRR de $58 \%$.

Após a interrupção da toma da metformina, os doentes foram observados durante uma a duas semanas e, nesse período, a incidência de diabetes aumentou de $25,2 \%$ para $30,6 \%$ no grupo previamente tratado com metformina e de $33,4 \%$ para $36,7 \%$ no grupo placebo. Quando 


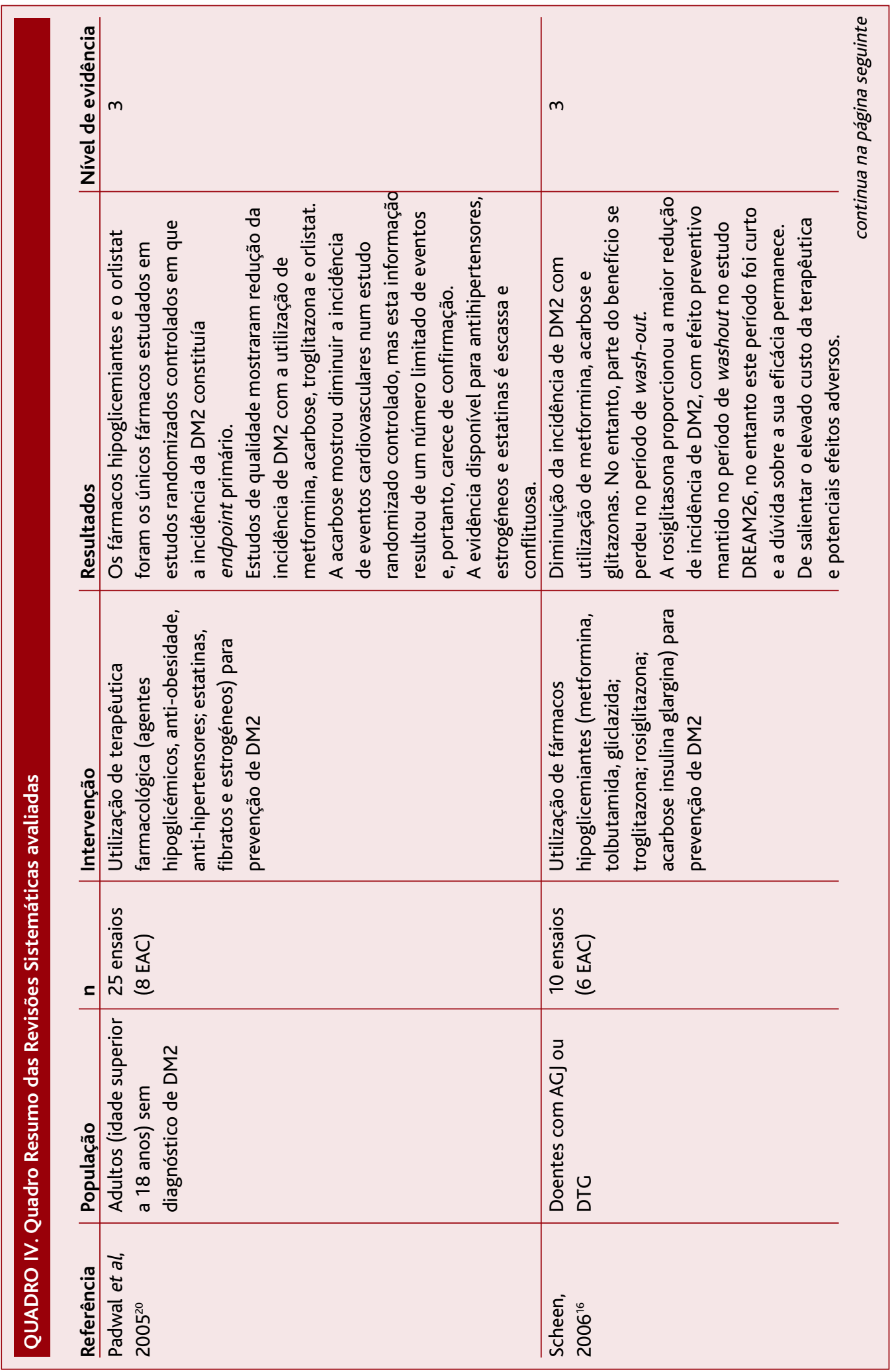




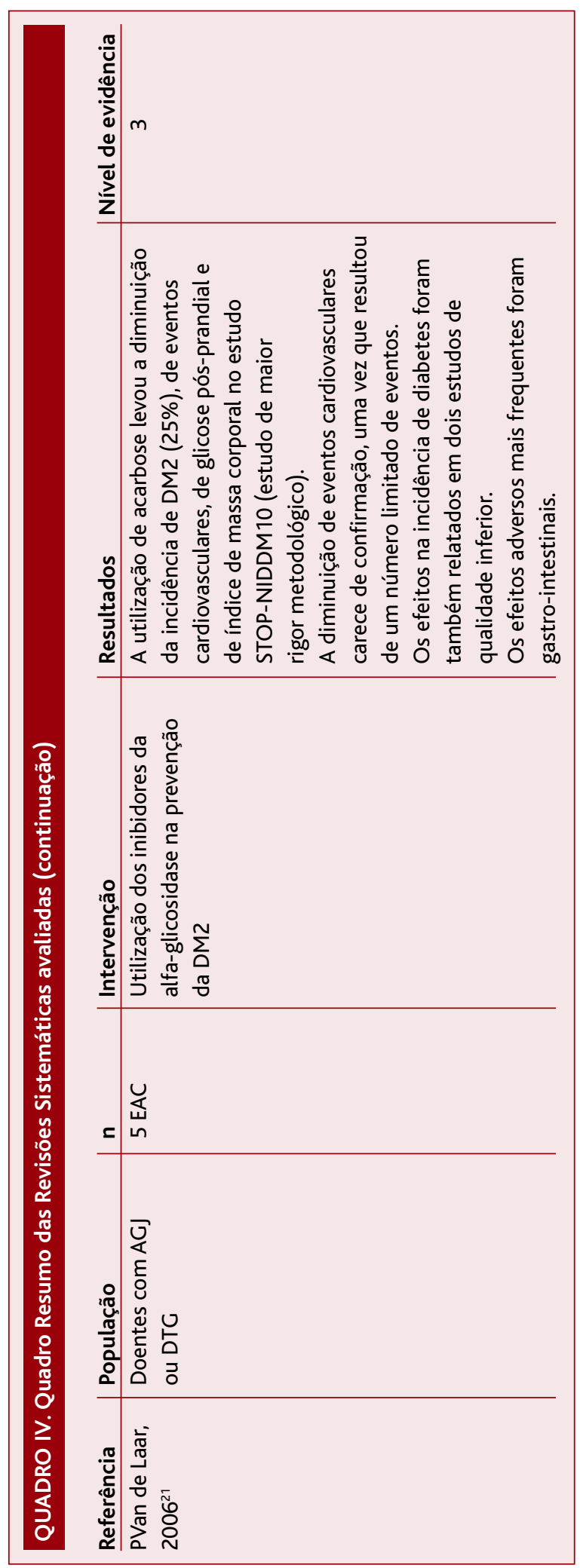

os resultados do período de wash-out foram incluídos na análise global, a redução da incidência de DM2 com a metformina ainda foi significativa (RRR de 25\%).

Os restantes estudos que incluíram a metformina ( $\mathrm{Li}$ et al, BIGPRO, Jarrett et al) não mostraram redução estatisticamente significativa na diminuição da incidência de DM2. No entanto, estes estudos tiveram incidências de DM2 muito reduzidas e teriam, provavelmente, pouco poder estatístico.

Atendendo a estes resultados, os autores não recomendam a metformina (ou qualquer outro fármaco, ver abaixo) para a prevenção da DM2. Realçam que parte do efeito da metformina desapareceu após um curto período de wash-out, não se podendo, desta forma, excluir um efeito residual de máscara. Para além disso, sendo a DM2 uma doença progressiva e tendo os estudos um seguimento relativamente curto, permanece por demonstrar se o efeito atribuído à metformina é um efeito verdadeiramente preventivo ou apenas um efeito de atraso da DM2 em um ou dois anos. Salientam, ainda, que permanecem por demonstrar reduções na morbilidade e mortalidade e benefícios em termos de custo-eficácia.

Na RS de Scheen, ${ }^{16}$ para além dos EAC avaliados por Padwal et al, ${ }^{20}$ foi também incluído o estudo Indian Diabetes Prevention Program (Indian DPP), no qual 531 doentes com DTG foram randomizados em quatro grupos: controlo, modificações do estilo de vida, metformina (250 b.i.d) e modificações do estilo de vida combinadas com metformina durante 3 anos. A incidência cumulativa de DM2 foi de 55\%, 39,3\%, 40,5\% e 39,5\%, respectivamente. Assim, no grupo tratado com intervenções no estilo de vida, a RRR de progressão para DM2 foi de 28,5\%; no grupo tratado com metformina foi de $26,4 \%$ e no grupo tratado com metformina mais intervenções no estilo de vida foi de $28,2 \%$. Neste estudo não se verificou, desta forma, efeito sinérgico entre as modificações do estilo de vida e a utilização de metformina. De realçar, contudo, que a dose utilizada de metformina foi apenas de $250 \mathrm{mg}$ b.i.d, muito inferior à utilizada no estudo DPP (850 mg b.i.d).

Ressalvando as questões também colocadas por Padwal et al, o autor conclui, contudo, que, atendendo à vasta experiência com este fármaco, ao seu baixo custo e boa tolerância, poderá ser uma opção válida para a prevenção da DM2 em doentes com risco muito ele- 


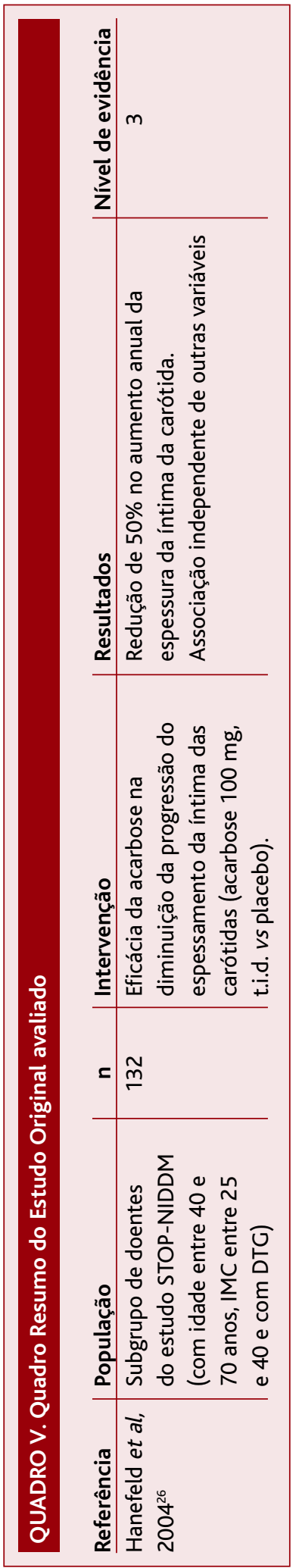

vado, com DTG, especialmente se jovens, obesos e familiares em primeiro grau de doentes com DM2.

\section{Acarbose}

Os inibidores da $\alpha$-glicosidase atrasam a absorção de carbohidratos e reduzem a glicemia pós-prandial inibindo a enzima $\alpha$-glicosidase, presente na bordadura em escova do intestino delgado, que hidrolisa os di e oligossacarídeos em monossacarídeos. A acarbose, embora quase não seja absorvida, tem propriedades de redução da insulinorresistência que são interpretadas como resultando da sua aç̧ão na redução da glicemia, ou seja, da redução da toxicidade de elevadas concentrações de glicose e diminuição da sobrecarga imposta às células $\beta$ para produção de insulina. Dos 3 inibidores da $\alpha$-glicosidase (miglitol, voglibose e acarbose), só a acarbose foi avaliada especificamente na capacidade de prevenir/atrasar a progressão de estádio de pré-diabetes para DM.

Foram encontradas 4 NOC e 3 revisões sistemáticas que avaliaram a utilização da acarbose em doentes pré-diabéticos.
Das 4 NOC seleccionadas, a NOC da ESC e EASD e a NOC do ICSI, no que concerne à prevenção da progressão para DM2, recomendam que doentes com elevado risco de DM2 devam receber aconselhamento para modificar os estilos de vida e, se necessário, terapêutica farmacológica, incluindo a metformina e a acarbose. A base da recomendação de utilização de acarbose em estádio de pré-diabetes centra-se numa RRR de progressão para DM2 de cerca de 25\%, encontrada no estudo Study to Prevent Non-Insulin Dependent Diabetes Mellitus (STOP-NIDDM).$^{10}$ A ADA e a AACE não recomendam a utilização de acarbose, uma vez que este fármaco se associa a importantes efeitos secundários gastrointestinais.

Em relação às revisões sistemáticas, Padwal et al, ${ }^{20}$ quanto à acarbose, incluiu apenas um EAC, o estudo STOP-NIDDM. Este estudo foi aquele que mostrou maior rigor metodológico. Incluiu uma amostra de 1.429 indivíduos com diminuição da tolerância à glicose, randomizados para acarbose (100 $\mathrm{mg}$ b.i.d.) $v$ s placebo, durante um período de 3,3 anos, mostrando uma diminuição da incidência de DM de $25 \%$. A utilização de acarbose levou, ainda, a uma maior probabilidade de reversão para normoglicemia e diminuição dos eventos cardiovasculares. No entanto, esta associação entre utilização de acarbose e diminuição do número de eventos cardiovasculares necessita de confirmação, uma vez que se baseou num número limitado de eventos. De salientar que, durante o período de wash-out do estudo (3 meses) houve perda do efeito. De referir também, a elevada taxa de descontinuação do estudo por parte dos indivíduos a receber acarbose $(31 \%$ vs $19 \%$ no grupo a receber placebo), atribuída aos efeitos secundários gastrointestinais do fármaco. Atendendo a estes resultados, os autores desta RS colocam as mesmas dúvidas já apontadas em relação à metformina e concluem que a acarbose não poderá ser recomendada para a prevenção da DM2.

Na revisão sistemática de Scheen, ${ }^{16}$ foi também incluído o estudo STOP-NIDDM. No entanto, o autor, apesar de salientar as mesmas dúvidas já colocadas por Padwal et al, considera que a acarbose pode ser uma alternativa em indivíduos com pré-diabetes e elevado risco cardiovascular.

Uma terceira revisão sistemática (de Van de Laar et $a l)$ incluiu na sua análise 5 EAC. ${ }^{21} \mathrm{O}$ estudo STOP- 
-NIDDM foi classificado como sendo aquele de maior rigor metodológico. Neste estudo a acarbose mostrou diminuir a incidência de DM em pacientes com diminuição da tolerância à glicose, facto que não pôde ser confirmado ou refutado por outros dois EAC, ${ }^{22,23}$ uma vez que não havia disponibilidade de dados. Outros 2 EAC menores e de inferior qualidade confirmaram a capacidade da acarbose reduzir a incidência de DM..$^{24,25}$ $\mathrm{O}$ autor concluiu que esta evidência deve ser tida em consideração perante um doente que poderá beneficiar de um tratamento activo da sua diminuição da tolerância à glicose.

Foi, ainda, seleccionado um estudo original que procurou avaliar a eficácia da acarbose na diminuição da progressão do espessamento da íntima das carótidas. ${ }^{26}$ Este estudo mostrou uma redução de cerca de $50 \%$ no aumento anual de espessura da íntima das carótidas. No entanto, uma vez que a amostra era constituída por um subgrupo de indivíduos do estudo STOP-NIDDM, um estudo que não incluiu esta avaliação como endpoint primário, esta associação deverá ser confirmada em futuros estudos.

\section{Tiazolidinedionas}

As tiazolidinedionas ou glitazonas (como são vulgarmente denominadas) são fármacos sensibilizadores da insulina com propriedades de redução da glicemia, muito utilizados na terapêutica da DM2. Estes agentes aumentam a sensibilidade à insulina tanto nos tecidos periféricos (músculo e gordura) como no fígado. Pensa-se que estes fármacos terão uma acção protectora da célula $\beta$ uma vez que melhoram a sua acção secretora.

Encontraram-se 4 NOC, 2 RS e 1 MA, com informação relativa à utilização desta classe de fármacos na prevenção de DM.

Apenas na NOC da ESC e EASD as glitazonas são apontadas como alternativa farmacológica na abordagem do doente com pré-diabetes e, destas, somente a rosiglitazona é referida. Esta recomendação tem como base o estudo Diabetes Reduction Assessment with Ramipril and Rosiglitazone Medication (DREAM), ${ }^{27}$ que mostrou uma RRR de progressão para DM de $61 \%$. Nas outras 3 NOC as glitazonas não constituem alternativa válida, uma vez que estiveram associadas, em vários EAC, a aumento de peso e aumento do número de casos de insuficiência cardíaca (rosiglitazona), sendo, tam- bém, uma classe de fármacos de preço elevado.

Encontrou-se uma metanálise que procurou estabelecer uma comparação entre a utilização de pioglitazona e rosiglitazona na pré-diabetes, mas os autores concluem que há poucos dados na literatura que permitam efectuar esta comparação. ${ }^{28}$ De salientar que, nesta metanálise, não foram incluídos EAC, posteriormente publicados, que avaliaram a utilização de pioglitazona e rosiglitazona em pré-diabéticos.

Em relação às RS, Padwal et al incluiu apenas um EAC, o estudo Troglitazone Prevention of Diabetes (TRIPOD).$^{29}$ Este estudo envolveu a participação de 236 mulheres com excesso de peso (IMC médio de 30,5 $\mathrm{kg} / \mathrm{m}^{2}$ ) e antecedentes de diabetes gestacional randomizadas para troglitazona ( $400 \mathrm{mg}$ b.i.d.) e placebo. Houve uma marcada redução da incidência de DM2, de $45 \%$ com placebo para $20 \%$ com troglitazona (RRR de $55 \%$ ). Este efeito foi observado após um período de 2,5 anos e ocorreu apesar de um aumento de peso modesto e estatisticamente não significativo de $0,3 \mathrm{Kg}$. A redução de risco de DM2 foi também robusta quando se consideraram apenas as mulheres com diminuição da tolerância à glicose no início do estudo (RRR de 49\%). Após o período de wash-out de 8 meses houve manutenção do efeito. No entanto, a elevada taxa de descontinuação do estudo (33\%) por efeitos secundários do fármaco é uma grande limitação. De salientar que a troglitazona foi retirada do mercado por estar associada a toxicidade hepática grave. Perante estes resultados, os autores não recomendam a utilização de glitazonas em doentes com pré-diabetes.

Na RS de Scheen, para além do EAC avaliado por Padwal et al, foram incluídos também mais 3 EAC. O estudo Pioglitazona in the Prevention of Diabetes (PIPOD) $^{30}$ foi um estudo metodologicamente muito semelhante ao estudo TRIPOD, mas com a utilização de pioglitazona $(30 \mathrm{mg} / \mathrm{dia}$ inicialmente e depois $45 \mathrm{mg} / \mathrm{dia}$ ) nas mulheres que completaram o estudo TRIPOD. Encontrou-se, também, uma redução marcada da incidência de DM2 com a utilização de pioglitazona.

O estudo DPP, já mencionado anteriormente, incluiu um braço onde foi avaliada a troglitazona ( $400 \mathrm{mg}$ b.i.d.) em relação ao placebo. Este braço foi abandonado por razões de segurança. No entanto, durante o período de utilização de troglitazona (0,9 meses) verificou-se uma 
incidência de DM2 de 3,0/100 pessoas-ano com este fármaco, comparado com 12,0; 6,7 e 5,1 casos/ 100 pessoas-ano no grupo placebo, metformina e alteração do estilo de vida, respectivamente $(\mathrm{p}<0,001$, troglitazona vs placebo; $\mathrm{p}=0,02$ troglitazona $v$ s metformina). Apesar destes resultados, verificou-se que 3 anos após a suspensão de troglitazona a incidência de DM2 era praticamente idêntica à do grupo placebo. Foi concluído que a troglitazona diminuiu a incidência de DM2 durante o seu período limitado de utilização, muito embora este efeito não se tenha mantido após suspensão.

O terceiro EAC incluído foi o estudo DREAM ${ }^{27}$ que, conforme já mencionado, comparou a utilização de rosiglitazona $v s$ placebo numa amostra de 5.269 indivíduos com AGJ ou DTG, tendo obtido uma substancial redução da incidência de DM2 (RRR de 61\%). De referir que este estudo incluiu, também, um braço onde foi avaliada a utilização de ramipril $v s$ placebo. Após o período de wash-out houve manutenção do efeito. Salienta-se a ocorrência de efeitos adversos como aumento de peso, retenção de fluidos e insuficiência cardíaca com a utilização de rosiglitazona, ainda que o estudo DREAM não tenha sido desenhado com o objectivo de avaliar resultados cardiovasculares.

Os autores concluem que, face a estes resultados, as dúvidas já colocadas anteriormente em relação à utilização de metformina e acarbose são as mesmas, isto é, será que existe realmente um efeito preventivo ou a utilização de glitazonas está apenas a mascarar ou atrasar o diagnóstico de DM2, ainda que os efeitos mais espectaculares de diminuição de incidência de DM2 tenham sido verificados com a utilização de glitazonas? Alertam, também, para os efeitos secundários desta classe de fármacos e para o seu preço elevado.

\section{Sulfonilureias e Metiglinidas}

Em relação às sulfonilureias e metiglinidas nenhuma NOC recomenda a sua utilização em doentes com pré-diabetes.

As RS de Padwal e de Scheen incluem apenas um EAC $^{31}$ recente, o estudo Fasting Hiperglicemia Study que procurou avaliar a utilização de gliclazida em indivíduos com pré-diabetes, não se tendo verificado diminuição da incidência de DM2 com a utilização deste fármaco. Além disso, a hipoglicemia surgiu em $3 \%$ dos participantes, mostrando ser um efeito secundário potencialmente limitativo. Scheen faz referência às metiglinidas como sendo um grupo de fármacos com um perfil farmacocinético que permite um melhor controlo da hiperglicemia pós-prandial, com menos riscos de hipoglicemia e que poderão representar uma alternativa às sulfonilureias. Encontra-se a decorrer um grande estudo que envolve a utilização de nateglinida e valsartan em doentes com diminuição da tolerância à glicose.

\section{Anti-Hipertensores}

No que diz respeito aos anti-hipertensores, para além do estudo DREAM que avaliou a utilização de ramipril $v s$ placebo em doentes pré-diabéticos e não encontrou diminuição da incidência de DM2, não há nenhum outro estudo que tenha avaliado como endpoint primário a incidência de DM2 e a evidência é insuficiente para recomendar uma determinada classe farmacológica em detrimento de outra.

\section{Orlistat}

A utilização do orlistat (inibidor da lípase gastroinestinal) na prevenção da DM2 foi avaliada em duas NOC e uma RS.

Em ambas as NOC (ADA e AACE), não se recomenda a utilização do orlistat atendendo ao seu custo e efeitos laterais e aos maiores benefícios demonstrados pelas alterações do estilo de vida e outros fármacos. ${ }^{14,18}$

$\mathrm{Na}$ RS de Padwal et al foi incluído um estudo. $\mathrm{O}$ Xenical in the Prevention of Diabetes in Obese Subjects (XENDOS) foi um EAC, com 4 anos de duração, no qual participaram 3350 doentes com IMC $\geq 30 \mathrm{~kg} / \mathrm{m}^{2}$ e com tolerância à glicose normal ou diminuída. Os participantes receberam intervenções sobre alterações do estilo de vida associados a terapêutica com orlistat (120 mg t.i.d.) ou com placebo. $\mathrm{O}$ orlistat reduziu a incidência de DM2 de 9 para 6\% (RRR de 37\%) e o peso em $2,8 \mathrm{~kg}$, comparativamente ao placebo. No entanto, $57 \%$ dos doentes descontinuaram o estudo e $91 \%$ dos doentes com orlistat referiram efeitos secundários gastrointestinais no primeiro ano de tratamento, comparativamente a $65 \%$ no grupo placebo. ${ }^{20}$

\section{CONCLUSÕES}

Vários estudos têm-se debruçado sobre o impacto de diversos fármacos na prevenção da diabetes mellitus. 
Uma maior atenção foi dirigida para os antidiabéticos orais (metformina, acarbose, tiazolidinedionas) e para os fármacos anti-obesidade (orlistat), encontrando-se, para estes fármacos, estudos que avaliaram a sua utilização na prevenção da diabetes como endpoint primário. De uma forma geral, existe uma diminuição da incidência da doença com a utilização de qualquer um deles, mas com resultados inferiores à aplicação intensiva de alterações de estilos de vida. Em relação a outros fármacos verifica-se que a informação é escassa ou de pior qualidade que a relacionada com os anteriores.

Um aspecto que foi abordado em muitos estudos é o facto de estes fármacos, ao diminuírem os valores da glicemia plasmática, estarem a mascarar um diagnóstico de diabetes mellitus tipo 2 , em vez de terem um real efeito preventivo. Esta é uma questão que não foi respondida em nenhum dos estudos analisados e que acompanha, também, a falta de demonstração da redução da morbilidade e mortalidade, que será realmente benéfica em doentes que não desenvolveram ainda a doença e que estejam a realizar estes tratamentos. É de salientar que a prescrição de princípios farmacológicos activos, para além do efeito esperado, pode ser acompanhada de efeitos secundários que diminuem a qualidade de vida dos doentes. Efeitos gastrointestinais adversos, risco de hipoglicemia, hepatotoxicidade ou mesmo aumento de eventos cardiovasculares foram reportados, em maior ou menor grau, nas diferentes classes farmacológicas e devem ser tidos em conta em qualquer prescrição racional, não esquecendo, também, o impacto económico para o doente e para a sociedade que a sua utilização acarreta. Esta revisão confirmou a utilidade de alguns fármacos anti-diabéticos numa prevenção orientada para a doença (redução dos valores de glicemia, atraso no diagnóstico analítico da diabetes) mas também encontrou falta de dados que abordem a utilização destes fármacos numa prevenção orientada para o paciente (redução da mortalidade, redução da morbilidade, melhoria da relação custo-eficácia) e que iriam reforçar os resultados obtidos.

Deste modo, a utilização de antidiabéticos orais e de fármacos anti-obesidade podem ser ponderados na abordagem de doentes com pré-diabetes (Força de Recomendação C), devendo ser sempre complementados com uma intervenção intensiva na modificação dos estilos de vida. Em 2006, a International Diabetes
Federation (IDF) realizou uma reunião de consenso com vários especialistas cujo objectivo foi a avaliação do impacto da diabetes tipo 2 e a necessidade de prevenção. Após a análise da evidência disponível (na forma dos estudos referidos ao longo desta revisão) um documento de consenso foi elaborado com recomendações sobre a prevenção da diabetes tipo 2 a nível populacional e individual. A nível populacional apela-se aos diversos governos à necessidade de promoção de estilos de vida saudáveis, usando, para tal, a comunicação social, implementando medidas fiscais e legislativas bem como medidas educacionais na comunidade, escolas e locais de trabalho. A nível individual, a estratégia aconselhada passa pela identificação das pessoas em mais alto risco de desenvolver a doença e, nestes casos, identificar indivíduos com diabetes não diagnosticada ou estados pré-diabéticos, beneficiando estes de intervenções para diminuir o risco da doença. A IDF preconiza, acima de qualquer medida, a implementação de alterações do estilo de vida, como o aumento da actividade física e a perda de peso e, ainda, a possibilidade de intervenção farmacológica salientado o uso da metformina, acarbose, agonistas PPAR-gama ou orlistat em doentes em que as alterações do estilo de vida não foram suficientes e, particularmente, em doentes idosos e obesos. ${ }^{32}$

Estão em curso estudos com novos fármacos e diferentes metodologias de investigação que podem fornecer mais dados na resolução desta problemática, mas serão necessários outros métodos (especialmente com a análise de evidência que interessa para o doente), que tragam maior qualidade a novos estudos, de forma a melhorar a força de recomendação.

\section{REFERÊNCIAS BIBLIOGRÁFICAS}

1. Wild S, Roglic G, Green A, Sicree R, King H. Global prevalence of diabetes: estimates for the year 2000 and projections for 2030. Diabetes Care 2004 May; 27 (5): 1047-53.

2. Direcção Geral de Saúde. Estudo da Prevalência da Diabetes em Portugal. Programa Nacional de Prevenção e Controlo da Diabetes. Direcção Geral de Saúde 2009. Disponível em: http://www.min-saude. pt/NR/rdonlyres/219DAD78-CD13-43CE-9221-42744B24176C/0/EstudoprevalenciaDiabetesemPortugal.pdf [acedido em 06/09/2009].

3. Roglic G, Unwin N, Bennett PH, Mathers C, Tuomilehto J, Nag S, et al. The burden of mortality attributable to diabetes: realistic estimates for the year 2000. Diabetes Care 2005 Sep; 28 (9): 2130-5.

4. Rao SS, Disraeli P, McGregor T. Impaired glucose tolerance and impaired fasting glucose. Am Fam Physician 2004 Apr 15; 69 (8): 1961-8.

5. Petersen JL, McGuire DK. Impaired glucose tolerance and impaired fas- 
ting glucose: a review of diagnosis, clinical implications and management. Diab Vasc Dis Res 2005 Feb; 2 (1): 9-15.

6. KnowlerWC, Barrett-Connor E, Fowler SE, Hamman RF, Lachin JM, Walker $E A$, et al. Reduction in the incidence of type 2 diabetes with lifestyle intervention or metformin. N Engl J Med 2002 Feb 7; 346 (6): 393-403.

7. Tuomilehto J, Lindström J, Eriksson JG, Valle TT, Hämäläinen H, IlanneParikka P, et al. Prevention of type 2 diabetes mellitus by changes in lifestyle among subjects with impaired glucose tolerance. N Engl J Med 2001 May 3; 344 (18): 1343-50.

8. Ramachandran A, Snehalatha C, Mary S, Mukesh B, Bhaskar AD, Vijay $\mathrm{V}$; the Indian Diabetes Prevention Programme. The Indian Diabetes Prevention Programme shows that lifestyle modification and metformin prevent type 2 diabetes in Asian Indian subjects with impaired glucose tolerance (IDPP-1). Diabetologia 2006 Feb; 49 (2): 289-97.

9. Pan XR, Li GW, Hu YH, Wang JX, Yang WY, An ZX, et al. Effects of diet and exercise in preventing NIDDM in people with impaired glucose tolerance: the Da Qing IGT and Diabetes Study. Diabetes Care 1997 Apr; 20 (4): 537-44.

10. Kosaka K, Noda M, Kuzuya T. Prevention of type 2 diabetes by lifestyle intervention: a Japanese trial in IGT males. Diabetes Res Clin Pract 2005 Feb; 67 (2): 152-62.

11. Chiasson JL, Josse RG, Gomis R, Hanefeld M, KarasikA, Laakso M; STOPNIDDM Trail Research Group. Acarbose for prevention of type 2 diabetes mellitus: the STOP-NIDDM randomised trial. Lancet 2002 Jun 15; 359 (9323): 2072-7.

12. Torgerson JS, Hauptman J, Boldrin MN, Sjöström L. XENical in the Prevention of Diabetes in Obese subjects (XENDOS) Study: a randomized study of orlistat as an adjunct to lifestyle changes for the prevention of type 2 diabetes in obese patients. Diabetes Care 2004 Jan; 27 (1):155-61.

13. Gerstein HC, Yusuf S, Bosch J, Pogue J, Sheridan P, Dinccag N, et al. Effect of rosiglitazone on the frequency of diabetes in patients with impaired glucose tolerance or impaired fasting glucose: a randomised controlled trial. Lancet 2006 Sep 23; 368 (9541): 1096-105.

14. American Diabetes Association. Standards of Medical Care in Diabetes - 2009. Diabetes Care 2009 Jan; 32 Suppl 1: S13-61.

15. Ebell MH, Siwek J, Weiss BD, Woolf SH, Susman J, Ewigman B, et al. Strength of recommendation taxonomy (SORT): a patient-centered approach to grading evidence in the medical literature. Am Fam Physician 2004 Feb 1; 69 (3): 548-56.

16. Scheen AJ.Antidiabetic agents in subjects with mild dysglycaemia: prevention or early treatment of type 2 diabetes? Diabetes Metab 2007 Feb; 33 (1): 3-12.

17. The Task Force on Diabetes and Cardiovascular Diseases of the European Society of Cardiology (ESC) and of the European Association for the Study of Diabetes (EASD). Guidelines on diabetes, pre-diabetes and cardiovascular diseases: full text. Eur Heart J 2007; 9 (Suppl C): C3-74.

18. American Association of Clinical Endocrinologists Diabetes Mellitus Clinical Practice Guidelines Task Force. AACE Diabetes Mellitus Guidelines for clicinal practice for the management of diabetes mellitus. Endocr Pract 2007 May-Jun; 13 Suppl 1: 1-68.

19. Institute for Clinical Systems Improvement. Diagnosis and management of type 2 diabetes mellitus in adults. Disponível em: http://www.guideline.gov [acedido em 06/10/2008].

20. Padwal R, Majumdar S, Johnson J, Varney J, McAlister FA. A systematic review of drug therapy to delay or prevent type 2 diabetes. Diabetes
Care 2005 Mar; 28 (3):736-44.

21. Van de Laar FA, Lucassen PLBJ, Akkermans RP, Van de Lisdonk EH, De Grauw WJ. Alpha-glucosidase inhibitors for people with impaired glucose tolerance or impaired fasting blood glucose. Cochrane Database Syst Rev 2006 Oct 18; (4): CD005061.

22. Holman RR, Blackwell L, Manley SE, Tucker L, Frighi V, Stratton IM. Sixyear results from the Early Diabetes Intervention Trial. Diabet Med 2003 Apr; 20 Suppl 2: S15.

23. Nijpels G, Boorsma W, Dekker JM, Kostense PJ, Bouter LM, Heine RJ. A study of the effects of acarbose on glucose metabolism in patients predisposed to developing diabetes: the Dutch acarbose intervention study in persons with impaired glucose tolerance (DAISI). Diabetes Metab Res Rev 2008 Nov-Dec; 24 (8): 611-6.

24. Fang YS, LiTY, Chen SY. Effect of medicine and non-medicine intervention on the outcomes of patients with impaired glucose tolerance: 5 year follow-up. Chin J Clin Rehabil 2004; 8 (30): 6562-3.

25. Wang $\mathrm{H}, \mathrm{XuWH}$, Wang GY.An evaluation on efficacy of Acarbose interfering treatment on IGT. Shanxi Clin Med J 2000; 9 (2): 116-7.

26. Hanefeld M, Chiasson JL, Koehler C, Henkel E, Schaper F, TemelkovaKurtschiev T. Acarbose slows progression of intima-media thickness of the carotid arteries in subjects with impaired glucose tolerance. Stroke 2004 May; 35 (5): 1073-8.

27. The DREAM (Diabetes Reduction Assessment with Ramipril and Rosiglitazone Medication) Trials Investigators. Effect of rosiglitazone on the frequency of diabetes in patients with impaired glucose tolerance or impaired fasting glucose: a randomized controlled trial. Lancet 2006 Sep 23; 368 (9541): 1096-105.

28. Norris SL, Carson S, Roberts C. Comparative effectiveness of pioglitazone and rosiglitazone in type 2 diabetes, prediabetes and the metabolic syndrome: a meta-analysis. Current Diabetes Rev 2007 May; 3 (2): $127-40$

29. Buchanan TA, Xiang AH, Peters RK, Kjos SL, Marroquin A, Goico J,et al. Preservation of pancreatic beta-cell function and prevention of type 2 diabetes by pharmacological treatment of insulin resistance in highrisk Hispanic women. Diabetes 2002 Sep; 51 (9): 2796-803.

30. Xiang AH, Peters RK, Kjos SL, Marroquin A, Goico J, Ochoa C, et al. Effect of pioglitazone on pancreatic $\beta$-cell function and diabetes risk in Hispanic women with prior gestational diabetes. Diabetes 2006 Feb; 55 (2): 517-22.

31. Herlihy OM, Morris RJ, Karunakaran S, Holman R. Sulphonylurea therapy over six years does not delay progression to diabetes. Diabetologia 2000 Aug; 43 Suppl. 1:A73.

32. Alberti KG, Zimmet P, Shaw J. International Diabetes Federation: a consensus on type 2 diabetes prevention. Diabet Med 2007 May; 24 (5): 451-63.

Os autores declararam não possuir conflitos de interesses

\section{ENDEREÇO PARA CORRESPONDÊNCIA}

António Macedo

Rua D. João de Castro, 225

4435-674 Baguim do Monte

E-mail: antmac.antonio@gmail.com

Recebido em 01/06/2009

Aceite para publicação em 03/11/2009 


\section{ABSTRACT}

\section{PHARMACOLOGIC THERAPY IN PREDIABETES}

Objectives: Type 2 Diabetes Mellitus (DM2) is a serious health problem associated with an increased morbidity, mortality and healthcare costs. It is often preceded by altered metabolic states as impaired fasting glucose (IFG) and/or impaired glucose tolerance (IGT). Lifestyle modification in patients with IGT showed a reduced incidence of DM2. Pharmacological therapy seems to prevent or at least delay the progression of DM2 in high risk patients, but doubts exist about their real benefit. The aim of this paper is to determine the real benefits in the use of pharmacological therapy in prediabetic states.

Data sources: A systematic review of articles in Portuguese, English or Spanish language, published between January 2008 and September 2008, was conducted in the medical databases National Guideline Clearinghouse, Bandolier, DARE, NHS and Medline using the keywords prediabetic state, prediabetes and therapeutics.

Methods of review: 103 articles were obtained. Those that compared the use of drugs in prediabetic states versus placebo or lifestyle change were included. SORT classification was used to attribute the level of evidence and strength of recommendation. Results: Nine articles were included in the review (4 guidelines, 1 meta-analysis, 3 systematic reviews and 1 randomized clinical trial). Some antidiabetic agents (metformin, acarbose and glitazones) and orlistat demonstrated a reduction in the risk of development of DM2 in pre-diabetic patients, remaining unclear if this benefit subsists after their withdrawal. Acarbose as also shown to reduce the progression of thickening of the intima layer in the carotid artery.

Conclusion: Although certain antidiabetic agents and orlistat have showed to reduce the risk of development of diabetes in pre-diabetic patients, the available evidence does not demonstrate patient oriented benefits with its use, since none of the studies evaluated morbidity, mortality or cost-effectiveness associated with the pharmacological therapy. Some studies that aim to clarify these issues are being conducted.

Keywords: Type 2 Diabetes Melitus; Prediabetic State; Therapeutics. 